The 47th IEEE International Midwest

\title{
Space-Time Coding for Ultra-Wideband OFDM-based Wireless System
}

\author{
Shing Tenqchen ${ }^{1 \varepsilon^{*}}$, Ying-Haw Shu ${ }^{1}$, Min-Chang, Sun ${ }^{1}$, Wu-Shiung Feng ${ }^{* *}$ \\ 'Lab 331, Department of Electrical Engineering, National Taiwan University, Taipei, Taiwan, R.O.C. \\ *Chunghwa Telecom Telecommunication Labs., 12, Lane 551, Sec. 5, \\ Min-Tsu Rd., Yang-Mei Zien, Tao-Yuan County, Taiwan 326, R.O.C. \\ **Department of Electronics Engineering, Chang Gung University, Taoyuan, Taiwan, R.O.C \\ E-mail: sto@chtcom.tw and paijusaprocomn.com.tw
}

\begin{abstract}
-we consider the performance analysis of beam space-time of a multiple-input multiple output (MIMO) orthogonal frequency-division multiplexing (OFDM) systems for high data rate wireless transmission. Employing multiple antennas at both the transmitter and receiver end offers a promising channel capacity, to facilitate the performance complexity tradeoff design in practice. This paper proposes the asymptotic capacity performance of Bezout WHT equalizers under i.i.d. AWGN and Rayleigh fading channels. Finally, we deploy our system with a high-data rate ultra multi-band OFDM (MB-OFDM) based wireless system for IEEE 802.15.3a.
\end{abstract}

\section{INTRODUCTION}

Currently, ultra-wideband (UWB) systems are gaining interests from researchers and companies due to its wireless link with high data rate [4]-[8]. The wireless indoor radio channel incurs a large number of constructive and destructive multipath components of the transported pulses at the receiver. However, the use of rake receivers, a diversity gain is achievable. Rake receivers consist of several baseband correlators or fingers which process the multipath components individually. Phases, amplitudes, and delays introduced by the channel are required for the Rake receivers and this can be solved by using a channel estimation algorithm to estimate these channel parameters.

Multi-band Orthogonal Frequency Division Multiplexing (MB-OFDM) has been standardized for a variety application, such as digital audio broadcasting (DAB) and terrestrial digital video broadcasting (DVB-T). The Basic idea of MBOFDM is that information is transmitted using OFDM modulation on each band. OFDM carriers are efficiently generated using a 128-point IFFT/FFT. Internal precision is reduced by limiting the constellation size to QPSK. Information bits are interleaved across all bands to exploit frequency diversity and provide robustness against multi-path and interference.

The main purpose of this paper is to simulate a UWB system based on pulsed multiband over the IEEE 802.15.3 UWB channel model [6] to observe the system's performance for a single user link with channel estimation algorithm. Here, we combine the Bezout inverse (zero-forcing) theory [2] and Walsh-Hadamard Transform (WHT) with the space-time coding (STC) techniques. Finally, via the maximum likelihood decoding rule analysis, it can be shown that the Bezout WHT outperforms the OFDM precoder in power, rate, and receiver implementation [2].

\section{PROBLEM DESCRIPTION}

Channel parameter estimation in UWB communications has been previously addressed in [3] \& [4] to assess the signal energy capture in Rake receivers as a function of the number of the Rake fingers. Figure 1 shows the block diagram of the MB-OFDM. The transmitted signal on each band is

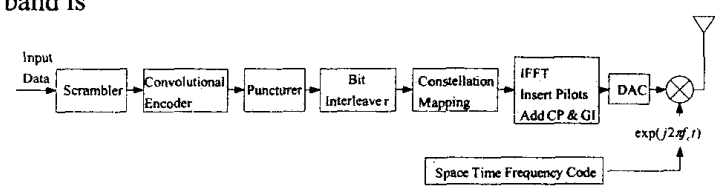

Fig. 1 Block diagram of Multiband-OFDM UWB[7]-[8].

For rates up to $200 \mathrm{Mb} / \mathrm{s}$, the input to the IFFT is forced to be conjugate symmetric (for spreading gains $\geq 2$ ). The analog section of TX can be simplified when the input is real. It is needed to only implement the "I" portion of DAC and mixer. For rates $>200 \mathrm{Mb} / \mathrm{s}$, need to implement full " $\mathrm{I} / \mathrm{Q}$ " transmitter. For rates $<200 \mathrm{Mb} / \mathrm{s}$ Only requires half the analog die size of a complete "I/Q" transmitter. The total of 128 tones consist of

-100 data tones used to transmit information (constellation: QPSK).

-12 pilot tones used for carrier and phase tracking.

-10 user-defined pilot tones.

- Remaining 6 tones including DC are NULL tones.

$-60.6 \mathrm{~ns}$ zero-padded cyclic prefix provides robustness against multi-path even in the worst channel environments.

- 9.5 ns guard interval provides sufficient time for switching between bands.

- Convolutional code of $\mathrm{R}=1 / 3, \mathrm{~K}=7$ with generator polynomial: $\quad g_{0}=\left[133_{8}\right], g_{1}=\left[145_{8}\right]$, $g_{2}=\left[175_{8}\right]$, puncturer \& depuncturer for $\mathrm{R}=3 / 4$.

- Bit interleaving is performed in 3 stages: symbol interleaver across OFDM, intra-symbol tone interleaver, intra-symbol cyclic shifts.

The signal transmission in a flat-fading MIMO system with $n_{T}$ transmit and $n_{R}$ receive antennas is modelled as:

$$
\mathbf{x}(k)=\mathbf{H s}(k)+\mathbf{n}(k)
$$


where $\mathbf{x}(\mathrm{k}) \in C^{n_{R}}, \mathbf{s}(k) \in C^{n_{T}}$ are sample stacks of complex-valued receiver data and source sequences, and $\mathbf{H}$ is the $n_{R} \times n_{T}$ channel transfer function.

$$
C_{Z F, i}=\log _{2}\left(1+\frac{\rho}{n_{T}\left\|H_{i}\right\|^{2}}\right),
$$

where $\rho=\frac{P_{T}}{N}$ is the nominal signal to noise ratio and the MIMO system is

$$
C_{Z F, i}=\log _{2}\left(1+\frac{\rho}{n_{T}}\left|\lambda_{i}\right|^{2}\right),
$$

where $\left\{\left|\lambda_{i}\right|^{2}\right\}_{i=1}^{n_{T}}$ are the singular values of $\mathbf{H}^{H} \mathbf{H}$ and the upper script of $H$ is the Hermittian transform.

Theorem 1: With a white space-time transmission distribution, the capacity per transmitted symbol for the timevarying, discrete-time, spatio-temporal channel $(J=S, V, M)$ subject to a transmitted-power limit for each channel use is

$$
\begin{aligned}
C & =\frac{1}{N} \sum_{k=0}^{N-1} I_{n_{k}} \\
& =E_{H}\left[W \log _{2}\left(\mathbf{I}+\chi_{2}^{2} \frac{P_{T}}{\sigma^{2}}\right)\right] \\
\mathrm{C} & =E_{H}\left[W \sum_{n=1}^{K} \log _{2}\left(I_{n_{k}}+\left|\lambda_{J, n}\right|^{2} \frac{P_{T}}{n_{T} \sigma^{2}}\right)\right] \\
\mathrm{C} & =E_{H}\left\{W \log _{2} \operatorname{det}\left[\left(\mathbf{I}+\frac{P_{T}}{\sigma^{2} n_{T}} Q\right)\right]\right\} \\
& =\frac{1}{N} \sum_{k=0}^{N-1} \log _{2}\left[\operatorname{det}\left(\mathbf{I}_{n_{R}}+S N R \mathbf{H}\left(e^{j 2 \pi(k / N)}\right) \cdot\left(\mathbf{H}^{\mu t}\left(e^{j 2 \pi(k / N)}\right)\right)\right]\right.
\end{aligned}
$$

where the quantity $I_{n_{k}}$ is the mutual information of the $k$ th MIMO OFDM subchannel. Note that since the bandwidth of each sub-channel and $P_{T}$ is the received signal power in the $i$ th sub-channel, $E_{H}[\cdot]$ denotes the expectation of channel $\mathbf{H}$ with respect to the random variable $\chi_{2}^{2}, \sqrt{\lambda_{J, n}}$ are the singular values of the channel matrix, $Q$ is defined as $Q=\left\{\begin{array}{l}\mathbf{H H}^{H}, n_{R}<n_{T} \\ \mathbf{H}^{H} \mathbf{H}, n_{R}>n_{r}\end{array}, \quad \mathbf{I}_{n_{R}}\right.$ is the identity matrix of size $n_{R}, \mathbf{H}^{\mathrm{k}}$ is an $n_{R} \times n_{T}$ channel matrix with its $(j, i)$-th entry $H_{j, i}^{t, k}$, and $S N R$ is the signal-to-noise ratio per receive antenna.

Proof Sketch: The proof follows directly from the definition of mutual information (Theorem 1) and the white transmitter- covariance matrix assumption. The reader is referred to [1],[5] for the complete proof.

Proposition 1: The distribution of $\mathbf{I}_{n_{k}}\left(n_{R}=0,1, \ldots, N-1\right)$ is independent of $k$ and given by

$I_{n_{R}} \approx \log \left[\operatorname{det}\left(\mathbf{I}_{n_{R}}+\rho \Lambda \mathbf{H}_{w} \mathbf{H}_{w}^{H}\right)\right]$, for $n_{\mathrm{R}}=0,1, \ldots, N-1$. where $\Lambda=\operatorname{diag}\left\{\lambda_{i}(\mathbf{R})\right\}_{i=0}^{n_{R}-1}, \mathbf{H}_{w}$ is an $n_{R} \times n_{T}$ i.i.d. random matrix with $\mathcal{C N}(0,1)$ entries, and $\mathbf{R}=\sum_{l=0}^{L-1} \mathbf{R}_{l}$.

Finally, $\lambda_{i}(\mathbf{R})$ denotes the $i$ th eigenvalue of $\mathbf{R}$.

Proof Sketch: The proof similarly follows directly from [1], [5]. The instantaneous channel capacity can be estimated by simulation.

3. Performance Analysis of STC-OFDM Systems

The MB-OFDM receiver shown at Fig.2 (a)-(c), is performed the OFDM-related FFT-aided demodulation of the incoming signal samples, followed by subcarrier-based channel transfer function equalization and dispreading with the aid of inverse WHT (IWHT) for the pre-select filter.

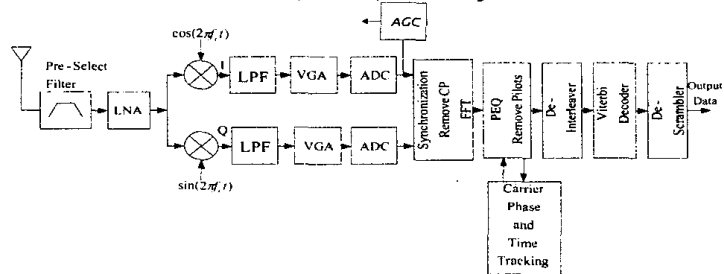

Fig. 2a The Pre-select Filter for IWHT STC-OFDM structure.

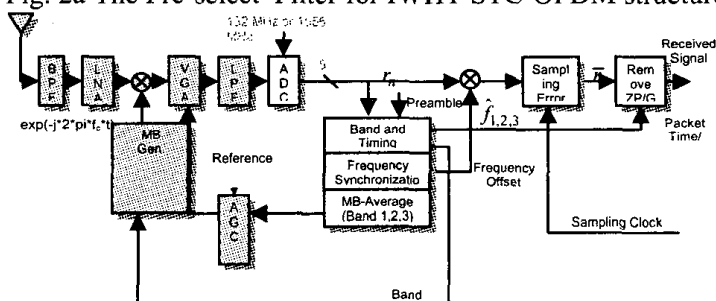

Fig.2b Block diagram of IWHT STC -OFDM receiver with synchronized sampling structure.

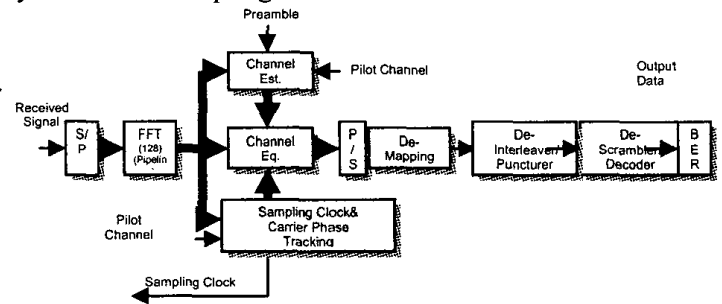

Fig. 2c Block diagram of IWHT STC -OFDM receiver with frequency offset estimation.

The symbol timing estimation is found by the precise estimate of the start of the band sequence of an incoming data 
and finding an approximate estimation of the start of the preamble of an incoming data packet. The typical algorithm is to use periodicity of preamble sequences to perform autocorrelation and using known preamble symbols with received symbols and find peak. After packet detection, the symbol timing algorithm refines the estimate to sample level precision. The refinement is performed by calculating the cross-correlation of the received signal $r_{n}$ and a known reference $s_{k}$, shown in Fig. 3(a)-(c).

Due to OFDM sensitive to carrier frequency offset, the frequency offset must be estimated and corrected before actual packets start. The typical algorithm is being used by the data aided algorithm: based on known preamble sequences and cyclic prefix based algorithm is to use the inherent structure of the OFDM signal provided by cyclic prefix or periodicity of preamble sequences. The structure is shown in Figure 3(c) [8]. The channel estimation is to use preamble sequences transmitted on every subcarrier and use pilot and decision-directed data to overcome time varying channel. After frequency offset compensation, there is always some residual frequency error to cause constellation rotation. The typical algorithm is the use of data-aided methods using pilot symbols. Twelve predefined subcarriers among the transmitted data are referred as pilots in 802.15.3a. The decision-directed method is used to improve the phase estimation quality.

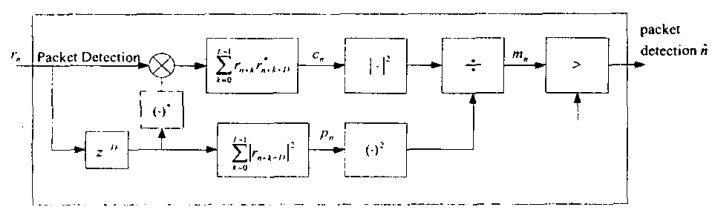

Fig. 3(a) Joint of band acquisition and packet detection (autocorrelation)

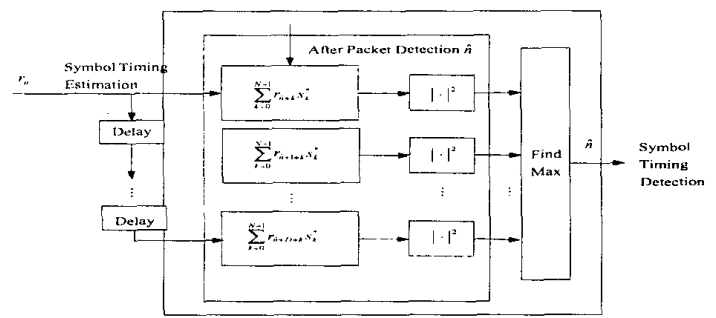

Fig. 3 (b) Symbol timing estimation (cross-correlation)

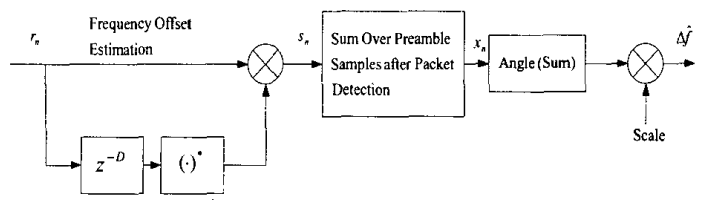

Fig. 3(c) The frequency offset estimation for the delay adjustment of IWHT STC-OFDM.

\section{Simulation Results}

This section shows the results of a number of simulations that are performed to obtain the results of IEEE 802.15.3a The main simulation parameters are based on the IEEE 802.15.3a standard and summarized in Table I, shown in Fig. 4 (a)-(f). Model simplifications/ assumptions for 802.15.3a are as follows:

Model assumptions:

- Baseband-equivalent model (no up/down conversion), Random data transmission (no data scrambling used)

- Fixed (selectable) number of data symbols per packet (no pad bits used), continuous frame-to-frame operation

- Fixed transmit power level; link-SNR specified (on-thefly), Idealized timing/frequency acquisition

- Not modeled:

- Other mandatory and optional data rates $(55,80,110$, $160,200,320,480 \mathrm{Mb} / \mathrm{s}$ )

- MAC/PHY interface and PLCP header (TX vector /RX vector, $\mathrm{HCS} / \mathrm{FCS}$ )

- Time windowing of OFDM symbols.

IEEE 802.15.3a Multiband OFDM - $200 \mathrm{Mb} / \mathrm{s}$ Mode [6]

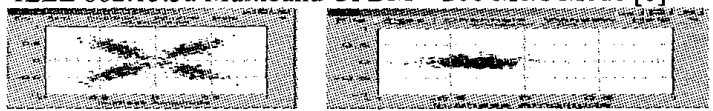

Fig. 4 (b) QPSK \& (c) QAM

The Simulation results of signal constellation of equalizer with QPSK \& QAM rate $R=1 / 3, K=7$ (Convolutional coding + puncturing) shown in Fig. 4(a)-(e).
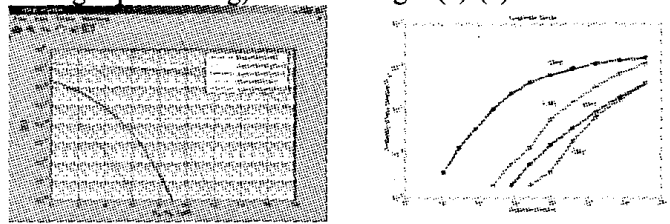

Fig. 4(d) The BER as a function of $E_{b} / N_{0}$ in AWGN \& Rayleigh channels with perfect parameters estimation. Fig.4(e) The results of system performance for prob. of miss detect (Pm) vs. distance @ $110 \mathrm{Mb} / \mathrm{s}$ was averaged over 500 noise realization for 100 channels in each channel environment.

\section{CONCLUDING REMARKS}

In this paper, we have considered the performance analysis, design criteria, and detection algorithm of MB-OFDM systems for high data-rate wireless transmission. The main contributions are listed as follows:

1) Multi-band OFDM system can achieve more than $10 \mathrm{~m}$ in all multipath channels. OFDM is a well-known multipath energy capture technique. Multi-band OFDM offers multi-mode scalable devices. IWHT STC-OFDM offers the best trade-off between the transceiver architectures and various parameters in receivers.

2) The bit-error probability is determined as a function of average channel SNR, with time-domain channel taps that fade with identical independent complex Gaussian 
distribution, deep frequency-domain interleaving, and ideal channel estimation.

\section{REFERENCES}

[1] T. L. Marzetta and B. H. Hochwald, "Capacity of a mobile multiple antenna communication link in Rayleigh flat fading," IEEE Trans. Inf. Theory, vol. 45, pp. 139-157, Jan 1999.

[2] S. Y. Kung, Y. Wu, and X, Zhang, "Bezout Space-Time Precoders and Equalizers for MIMO Channels," IEEE Trans. Signal Processing, vol. 50, pp.2499-2514, Oct. 2002.

[3] V. Lottici, A. D'Andrea, U. Mengali, "Channel Estimation for Ultra-wideband Communications," IEEE Trans. on Selected Areas in Communications, vol. 20, no. 9, pp. 1638-1645, Dec. 2002

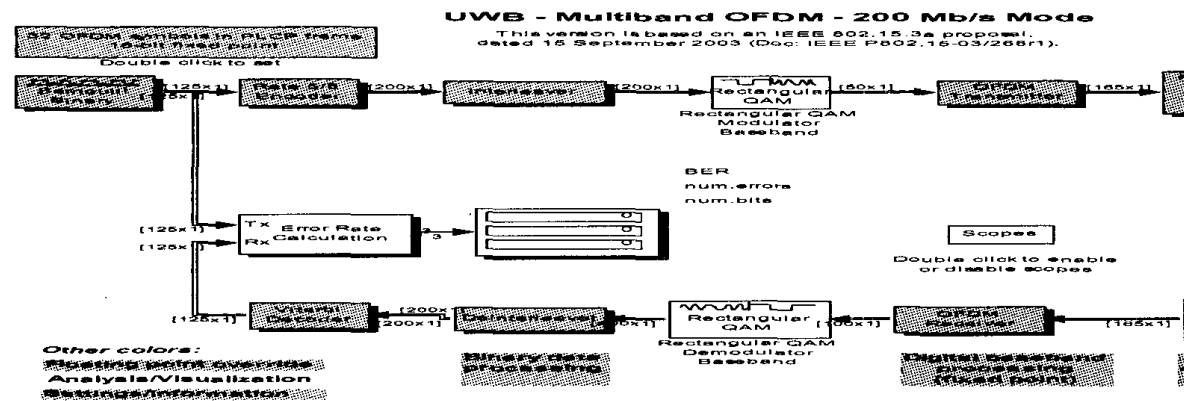

4] J. Balakrishnan, A. Batra and A. Dabak, "A multiband OFDM system for UWB communication," IEEE Ultra Wideband Systems and Technologies Conf. pp.354-358, Nov. 2003.

[5] H. Bolcskei, D. Gesbert, and A. J. Paulraj, "On the capacity of OFDM-based spatial multiplexing systems," IEICE Trans. On Comms., No. 2, pp. 225-234, Feb. 2002.

[6] http//grouperiece org/groups/802/15/pub/2003/Mar03/ 02490r1P802-15_SG3a-Channel-ModelingSubcommittee-report-Final.zip.

[7] A. Batra et. al., "Multi-band OFDM physical layer proposal for IEEE 802.15 task group 3a", IEEE 802.1503/268r2, TI Inc., 10 Nov., 2003.

[8] J. H. Deng, "Performance Evaluation and Architecture Design for Multiband OFDM UWB System using MATLAB", MATLAB Simulink Terasoft Conf. at Taipei June 1 \& 2, 2004

Fig. 4 (a) The Simulink model of UWB - Multiband OFDM 200Mb/s Mode.

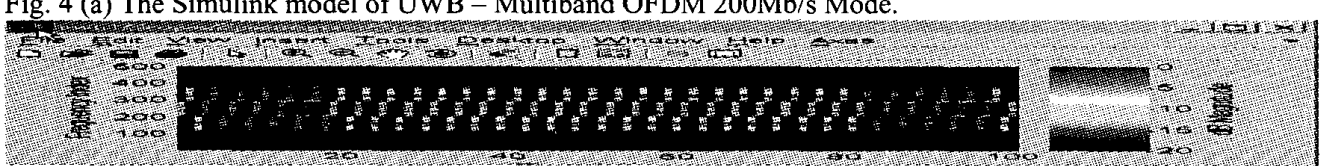

Fig. 4(f) The Simulation result of spectral gram of UWB - Multiband OFDM 200Mb/s Mode.

\begin{tabular}{|l|l|l|l|l|l|l|l|}
\hline Info. Data Rate & $55 \mathrm{Mbps}$ & $80 \mathrm{Mbps}$ & $110 \mathrm{Mbps}$ & $160 \mathrm{Mbps*}$ & $200 \mathrm{Mbps}$ & $320 \mathrm{Mbps} * *$ & $480 \mathrm{Mbps}^{* *}$ \\
\hline Modulation/Constellation & OFDM/QPSK & FFDM/QPSK & OFDM/QPSK & OFDM/QPSK & OFDM/QPSK & OFDM/QPSK & OFDM/QPSK \\
\hline FFT Size & 128 & 128 & 128 & 128 & 128 & 128 & 128 \\
\hline Coding Rate (K=7) & $\mathrm{R}=11 / 32$ & $\mathrm{R}=1 / 2$ & $\mathrm{R}=11 / 32$ & $\mathrm{R}=1 / 2$ & $\mathrm{R}=5 / 8$ & $\mathrm{R}=1 / 2$ & $\mathrm{R}=3 / 4$ \\
\hline Spreading Rate & 4 & 4 & 2 & 2 & 2 & 1 & 1 \\
\hline Information Tones & 25 & 25 & 50 & 50 & 50 & 100 & 100 \\
\hline Data Tones & 100 & 100 & 100 & 100 & 100 & 100 & 100 \\
\hline Info. Length & $242.4 \mathrm{~ns}$ & $242.4 \mathrm{~ns}$ & $242.4 \mathrm{~ns}$ & $242.4 \mathrm{~ns}$ & $242.4 \mathrm{~ns}$ & $242.4 \mathrm{~ns}$ & $242.4 \mathrm{~ns}$ \\
\hline Cyclic Prefix & $60.6 \mathrm{~ns}$ & $60.6 \mathrm{~ns}$ & $60.6 \mathrm{~ns}$ & $60.6 \mathrm{~ns}$ & $60.6 \mathrm{~ns}$ & $60.6 \mathrm{~ns}$ & $60.6 \mathrm{~ns}$ \\
\hline Guard Interval & $9.5 \mathrm{~ns}$ & $9.5 \mathrm{~ns}$ & $9.5 \mathrm{~ns}$ & $9.5 \mathrm{~ns}$ & $9.5 \mathrm{~ns}$ & $9.5 \mathrm{~ns}$ & $9.5 \mathrm{~ns}$ \\
\hline Symbol Length & $312.5 \mathrm{~ns}$ & $312.5 \mathrm{~ns}$ & $312.5 \mathrm{~ns}$ & $312.5 \mathrm{~ns}$ & $312.5 \mathrm{~ns}$ & $312.5 \mathrm{~ns}$ & $312.5 \mathrm{~ns}$ \\
\hline Channel Bit Rate & $640 \mathrm{Mbps}$ & $640 \mathrm{Mbps}$ & $640 \mathrm{Mbps}$ & $640 \mathrm{Mbps}$ & $640 \mathrm{Mbps}$ & $640 \mathrm{Mbps}$ & $640 \mathrm{Mbps}$ \\
\hline Multi-path Tolerance & $60.6 \mathrm{~ns}$ & $60.6 \mathrm{~ns}$ & $60.6 \mathrm{~ns}$ & $60.6 \mathrm{~ns}$ & $60.6 \mathrm{~ns}$ & $60.6 \mathrm{~ns}$ & $60.6 \mathrm{~ns}$ \\
\hline
\end{tabular}

Table I: Simulation parameters, Based on the IEEE 802.15.3a Multi-band OFDM 55 480Mb/x Mode. 